\title{
HOW BUILDING ADJACENCY AFFECTS OCCUPANT-PERCEIVABLE VIBRATIONS DUE TO URBAN SOURCES: A PARAMETRIC STUDY
}

\author{
Peter Persson $^{1}$, Loukas F. Kallivokas ${ }^{2}$, Lars V. Andersen ${ }^{3}$, Andrew T. Peplow ${ }^{4}$ \\ ${ }^{1}$ Department of Construction Sciences, Lund University \\ P.O. Box 118, SE-221 00 Lund, Sweden \\ e-mail: peter.persson@ construction.lth.se \\ ${ }^{2}$ Department of Civil, Architectural and Environmental Engineering, The University of Texas at Austin \\ Austin, TX 78712-1068, USA \\ e-mail: loukas@mail.utexas.edu \\ ${ }^{3}$ Department of Engineering, Aarhus University \\ 8000 Aarhus C, Denmark \\ e-mail: lva@eng.au.dk \\ ${ }^{4}$ Department of Natural Sciences and Public Health, Zayed University \\ P.O. Box 144534, Abu Dhabi, United Arab Emirates \\ e-mail: andrew.peplow@zu.ac.ae
}

Keywords: Building Vibrations, Building Adjacency Effects, Ground Vibrations, Coupled FEM, Frequency Domain, Parametric Study

Abstract. Urban densification, despite the economic and social opportunities it offers, brings with it challenges to the well-being of citizens. A direct effect of densification is the rise in noiseand vibration-producing sources, which can lead to both health and comfort issues. In determining the risk associated with excessive vibration levels, the distance between the source and the receiver or target building, the ground properties, and the building type, are all important factors. In this paper, we are concerned with the effect that adjacent buildings have on the prediction of the vibration levels in a residential building, caused by external ground surface loads. The parametric studies were conducted using a coupled finite element model of the ground and the buildings. The analyses were conducted in the frequency domain, and vibrational velocity levels were computed at the receiver building in order to assess how building adjacency affects the receiving building's vibrational response. 


\section{INTRODUCTION}

The densification of urban areas, driven mostly by economic forces, continues unabated. Urban densification, despite the economic and social opportunities it offers, brings with it challenges to the well-being of citizens. A direct effect of densification is the rise in noise- and vibration-producing sources, which can lead to vibrations that exceed human-tolerable levels. Current construction trends, which tend to favor the use of lighter materials (e.g., timber), promote higher levels of human-perceivable vibrations. In addition, in dense urban settings, the proliferation of vibration-producing sources has an effect on vibration-sensitive equipment, whether in hospitals, research laboratories, or industrial facilities, that cannot be ignored.

Of the many different vibration sources, of interest herein is the analysis of traffic-induced building vibrations, since it remains one of the dominant sources of vibrations in urban environments, further aggravated by densification. The traffic-induced vibration analysis is demanding, since the problem involves a large number of difficult-to-estimate parameters. In broad strokes, the problem can be divided into the three different parts illustrated in Fig. 1: the external source; the medium; and the receiver. The parameters and the properties of each part affect the vibration levels in the receiver building. In devising numerical models to estimate the vibration levels, adjacent buildings are usually not taken into account-it is common practice to only include the receiving building within the model. The effect of adjacent buildings in the response of a receiver building has been primarily studied in the context of earthquake engineering, the socalled structure-soil-structure interaction and/or "site-city effects", where the presence of adjacent buildings can significantly alter the response dynamics (see, for example, [1, 2]). However, the influence of these interaction effects is not a well-studied area for traffic-induced building vibrations. Exceptions include the study of the effect of structural modifications to the receiving building and its surroundings in order to mitigate the vibrational response induced by ground sources [3, 4, 5, 6, 7, 8].

In the present study, we investigate the effect of structural modifications to an adjacent building on the receiving building's vibrational response due to ground surface loading. An example of a suitable and commonly-arising application is the study of traffic-induced building vibrations were residents may be complaining due to increased vibrational levels. In Section 2 we describe modeling of externally induced building vibrations, and in Section 3 we discuss the developed computational model. Section 4 presents the conducted parametric study and the numerical results. In Section 5 we discuss pertinent conclusions of the study.

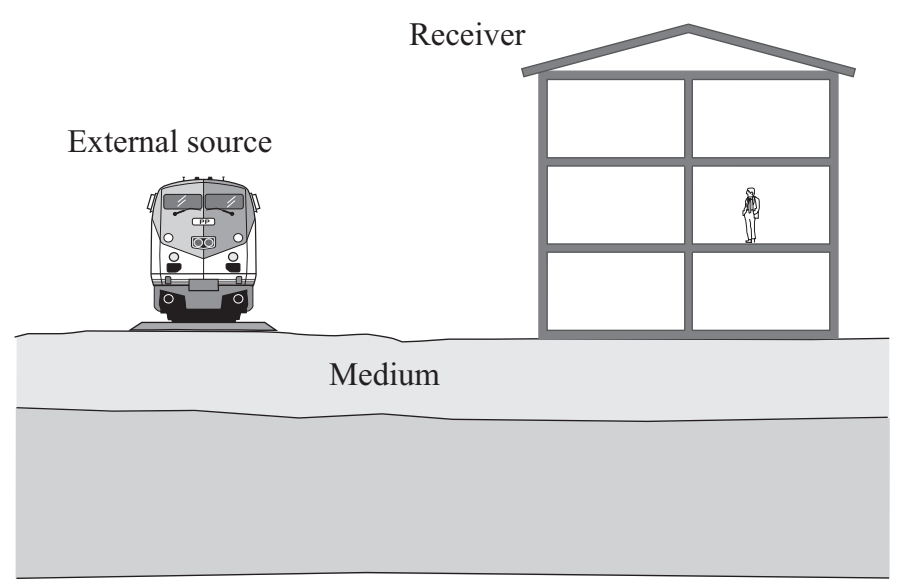

Figure 1: Illustration of typical components of a building vibration problem in the built environment. 


\section{MODELING OF EXTERNALLY INDUCED BUILDING VIBRATIONS}

The modeling procedure used in the present study is based on the assumption of linear elastic behavior, since we consider only low-amplitude vibrations induced by, for example, train and/or road traffic. The analyses of the dynamic loading is performed in the frequency domain and the finite element (FE) method is used to compute velocity levels. Since the spatial variability of the soil and of the building properties occur at scales much smaller than the wavelengths that correspond to the frequency range of interest, in additional to linearity, we assumed homogeneous and isotropic material behavior throughout.

After spatial discretization, the FE model of a linear dynamic system provides a semi-discrete equation of motion in the form of the following system of ordinary differential equations:

$$
\mathbf{M u ̈}+\mathbf{C u}+\mathbf{K u}=\mathbf{f},
$$

where $\mathbf{M}, \mathbf{C}$, and $\mathbf{K}$ denote the mass, damping, and stiffness matrices, respectively, whereas $\mathbf{u}$ denotes the displacements and $\mathbf{f}$ the loads. Dots indicate derivatives with respect to time. In case of steady state displacement response to harmonic excitation, $\mathbf{u}$ and $\mathbf{f}$ can be expressed as complex harmonic functions:

$$
\mathbf{f}=\hat{\mathbf{f}} \mathrm{e}^{\mathrm{i} \omega t}, \quad \mathbf{u}=\hat{\mathbf{u}} \mathrm{e}^{\mathrm{i} \omega t}
$$

where $\hat{\mathbf{f}}$ and $\hat{\mathbf{u}}$ denote the complex load amplitude and the displacement amplitude, respectively, i denotes the imaginary unit, $\omega$ denotes the angular frequency, and $t$ denotes time. Introducing Eq. (2) in the equation of motion (Eq. 1) yields the equation of motion in the frequency domain:

$$
\mathbf{D}(\omega) \hat{\mathbf{u}}=\hat{\mathbf{f}},
$$

where the dynamic stiffness matrix $\mathbf{D}(\omega)$ can be expressed as:

$$
\mathbf{D}(\omega)=-\omega^{2} \mathbf{M}+\mathrm{i} \omega \mathbf{C}+\mathbf{K} .
$$

We used a rate-independent (hysteretic) damping model, and thus, the damping can be described by a loss factor [9], as in:

$$
\mathbf{K} \eta=\omega \mathbf{C} .
$$

Inserting Eq. (5) into Eq. (4), we arrive at:

$$
\mathbf{D}(\omega)=-\omega^{2} \mathbf{M}+(1+\mathrm{i} \eta) \mathbf{K} .
$$

The imaginary part of the stiffness matrix is often referred to as the structural damping matrix. Equation (6) was used to model both the structures and the soil. We note that for the soil, the linear elastic model accounts for the bulk compressional and shear waves, as well as for any interface waves (Rayleigh on the free surface, and Stoneley along any interfaces between soil layers). As is often the case, the FE model occupies a finite computational domain, which is truncated via the introduction of suitable absorbing boundaries. Here, to reduce the computational effort, we used dashpots [10], which are effective when waves impinge at normal incidence. 


\section{COMPUTATIONAL MODEL}

The coupled FE model comprises both the soil and the building structures, which are fully coupled. See Fig. 2 for a schematic of the model. Plane strain conditions were applied, thus a line load as well as an elongated building exposed to planar waves were assumed.

The soil in the example case consists of a stiff $10 \mathrm{~m}$ deep soil layer resting on top of bedrock-conditions common in the south of Sweden; see Fig. 3 for the Youngs modulus E, Poissons ratio $\nu$, mass density $\rho$, and the loss factor $\eta$ for the soil materials. Treating the bedrock as a linear elastic material rather than a rigid foundation is an important parameter in this study. The dimensions of the building are shown in Fig. 2. The building structure consists of $200 \mathrm{~mm}$ thick concrete walls and $300 \mathrm{~mm}$ thick concrete slabs. The reinforcing bars are assumed to not influence the response; the isotropic material properties used for the concrete are given in Table 1. The model is excited by a vertical harmonic unit load applied on the ground surface $50 \mathrm{~m}$ from the receiver building; the load frequency was varied between 1 and $80 \mathrm{~Hz}$ in steps of $0.5 \mathrm{~Hz}$. The frequency range was set to relate to human response, on the basis of the ISO standard [12].

Abaqus 6.13 [11] was used to compute the coupled response. To ensure that the FE analyses provide results of adequate accuracy, the influence of various parameters such as the aspect ratio of the finite elements, the size of the computational domain, and the reflection of waves at artificial boundaries have been studied, while the mesh density was adjusted to account for typical minimum number of nodes per wavelength criteria. The soil, as well as the buildings, were modeled with eight-node (serendipity) plane strain elements. Given the materials and fre-

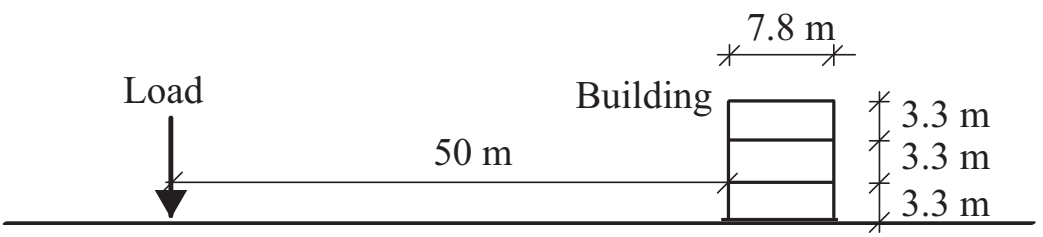

Ground

Figure 2: Schematic of the model. Center-to-center dimensions are also shown.

\begin{tabular}{|c|c|c|c|}
\hline I & Soil & $E=500 \mathrm{MPa}$ & \\
\hline i & & $v=0.48$ & \\
\hline I & & $\rho=2000 \mathrm{~kg} / \mathrm{m}^{3}$ & $10 \mathrm{~m}$ \\
\hline I & & $n=0.10$ & \\
\hline I & & & \\
\hline I & & & \\
\hline I & Bedrock & $E=10000 \mathrm{MPa}$ & \\
\hline I & & $v=0.40$ & \\
\hline I & & $\rho=2500 \mathrm{~kg} / \mathrm{m}^{3}$ & \\
\hline I & & $n=0 \Omega 4$ & \\
\hline I & & $\eta=0.04$ & \\
\hline I & & & \\
\hline
\end{tabular}

Figure 3: Schematic of the ground profile; inserts describe the ground material properties. 


\begin{tabular}{cc}
\hline Property & Value \\
\hline Young's modulus & $32 \mathrm{GPa}$ \\
Poisson ratio & 0.2 \\
Mass density & $2500 \mathrm{~kg} / \mathrm{m}^{3}$ \\
Loss factor & 0.04 \\
\hline
\end{tabular}

Table 1: Material properties used for concrete.

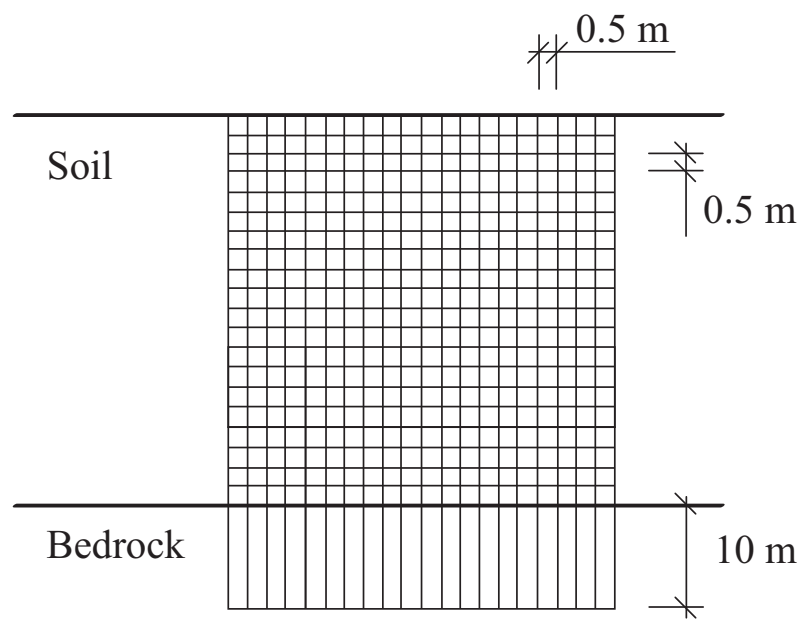

Figure 4: Schematic of the soil element mesh.

quency range of interest, the mesh convergence study resulted in an element size of $0.5 \times 0.5 \mathrm{~m}^{2}$ for the soil layer and $0.5 \times 10 \mathrm{~m}^{2}$ for the bedrock. Generally, an element size of $0.3 \times 0.3 \mathrm{~m}^{2}$ was used for the slabs, and $0.2 \times 0.3 \mathrm{~m}^{2}$ for the walls. The FE mesh of the soil is shown in Fig. 4 .

\section{PARAMETRIC STUDY}

In order to investigate the effects that various parameters of the adjacent building have on the receiving building's vibration response, a single parameter at a time was varied while the others were kept constant. In total, five different parameters related to the adjacent building were varied, and the results are presented in the following five subsections 4.1-4.5. Specifically, we report on the effect of:

1. Distance between the buildings

2. Size of adjacent building footprint

3. Adjacent building cellar depth

4. Adjacent building mass

5. Adjacent building damping

In computing one value representing the vibration response we want to use a metric that is sensitive to the peaks in the response spectra. We are therefore using a Root Mean Quad value (RMQ), calculated as follows:

$$
\mathrm{RMQ}=\frac{1}{n}\left(\sum|V|_{j}^{4}\right)^{1 / 4}
$$




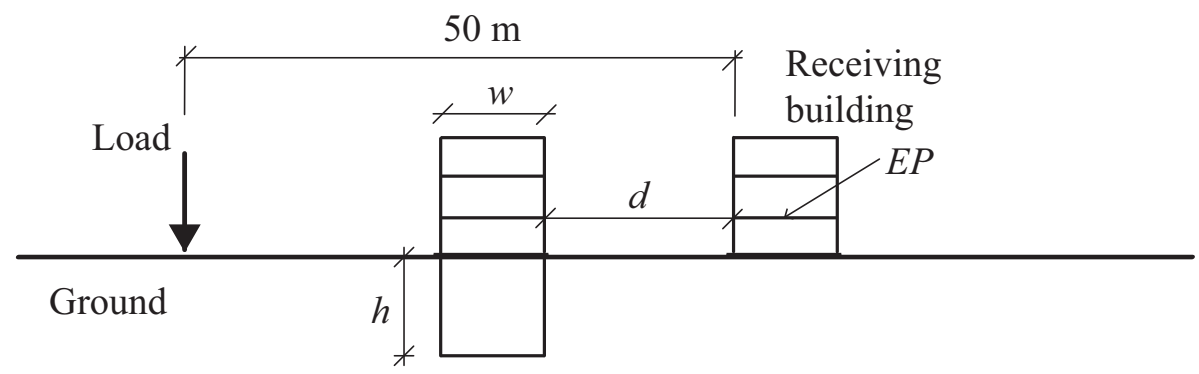

Figure 5: Setup with adjacent building in front of receiving building.

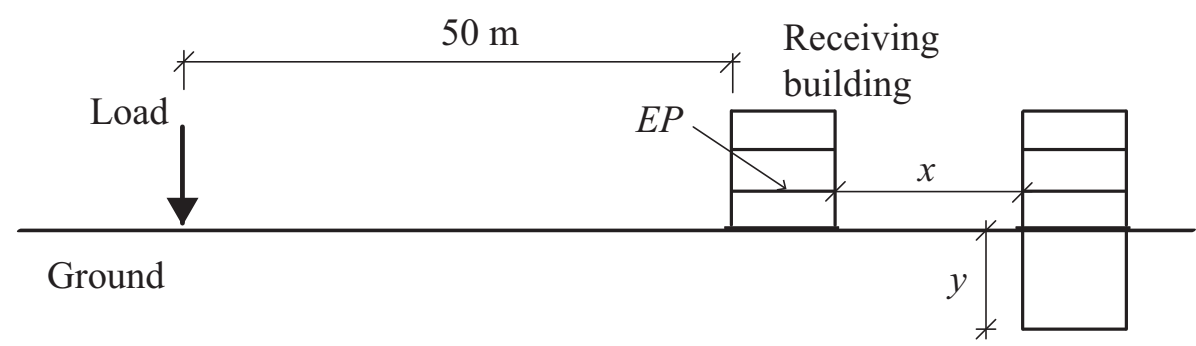

Figure 6: Setup with adjacent building behind receiving building.

where $j$ denotes each discrete frequency, and $n$ is the total number of frequencies. The vertical velocities, $|V|$, were evaluated as complex magnitudes at the mid-point of the first floor level; the specific locations are indicated in Figs. 5 and 6 as EP (Evaluation Point).

\subsection{Distance between the buildings}

In this subsection we evaluate to what extent the distance between the buildings affects the vibrations in the receiving building.

First we consider the case when the adjacent building $(h=0, w=8 \mathrm{~m})$ is located in front of the receiving building as seen in Fig. 5. Figure 7 shows that having an adjacent building between the vibration source and the receiving building affects the response significantly. It also shows that the gap-distance $d$ has a minor effect on the response, and that no clear trend can be seen.

Secondly, we consider the case of having an adjacent building without a cellar $(h=0)$ located behind the receiving building. The calculations showed that the response was not affected to any appreciable extent, independently of the distance $x$ between the buildings (cf. Fig. 6). Therefore, we do not show the corresponding frequency response. However, if an $8 \mathrm{~m}$-deep cellar is considered, i.e. $y=8 \mathrm{~m}$, the response in the receiving building is affected. The effect of various distances, $x$, between the buildings for this case is presented in Fig. 8. As seen in the figure, there is an effect on the response depending on the distance to the adjacent building. The effect on the spectrum is rather small but since it affects the large frequency peaks, it still results in a considerable change in the evaluated metric of the response. However, no apparent general trends can be seen other than that the distance between the buildings is of importance. 


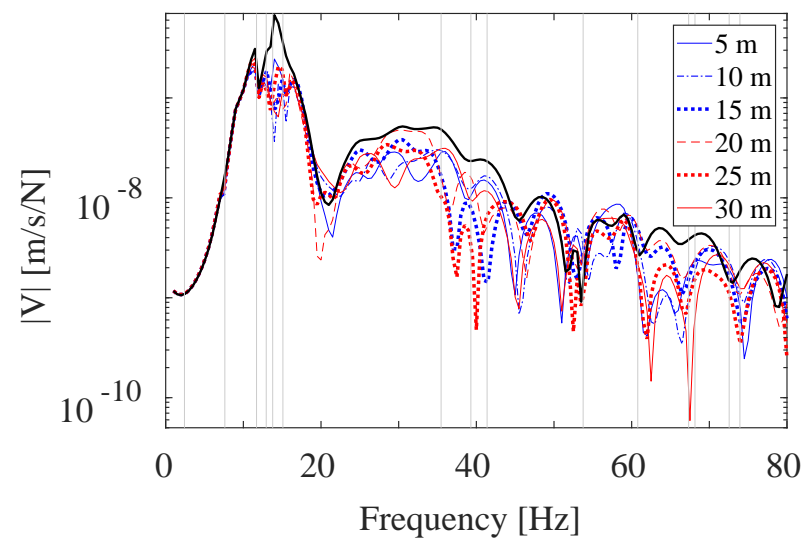

(a) Frequency spectrum for tested values of $d$.

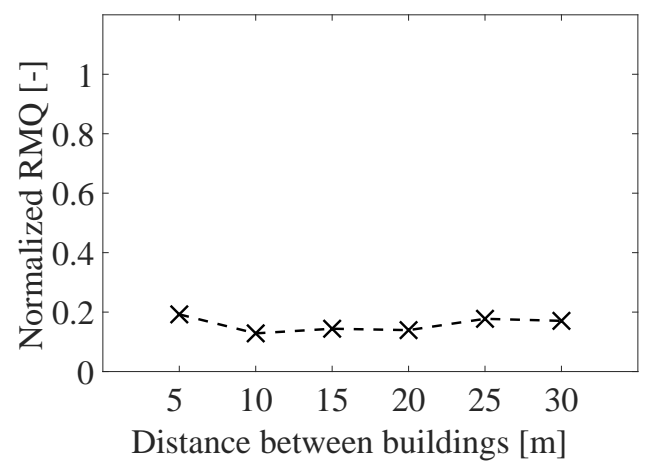

(b) RMQ value normalized to the reference case.

Figure 7: Vibration levels computed with the adjacent building in front of the receiving building. The distance $d$ is being varied. A setup with no adjacent building represents the reference case. In the left subfigure, the solid black line represents the reference case and the gray vertical lines show the receiving building's eigenfrequencies.

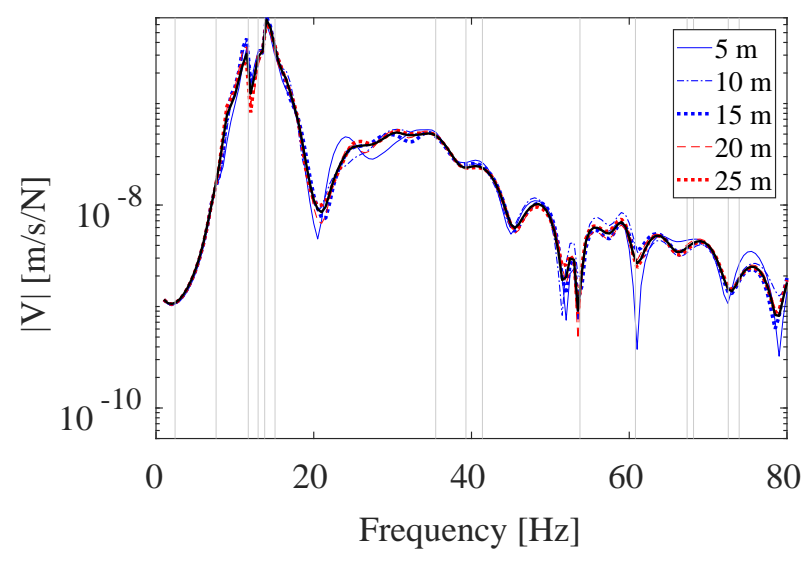

(a) Frequency spectrum for the tested values of $x$.

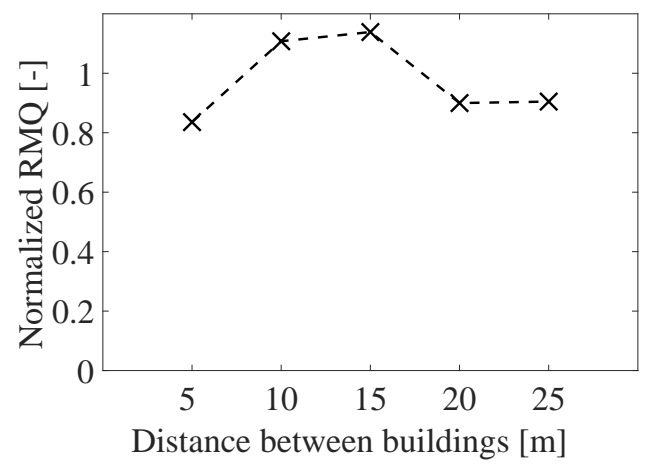

(b) RMQ value normalized to the reference case.

Figure 8: Vibration levels computed with the adjacent building having $8 \mathrm{~m}$ cellar behind the receiving building. The gap-distance $x$ being varied. A setup with no adjacent building represents the reference case. In the left subfigure, the solid black line represents the reference case and the gray vertical lines show the receiving building's eigenfrequencies. 


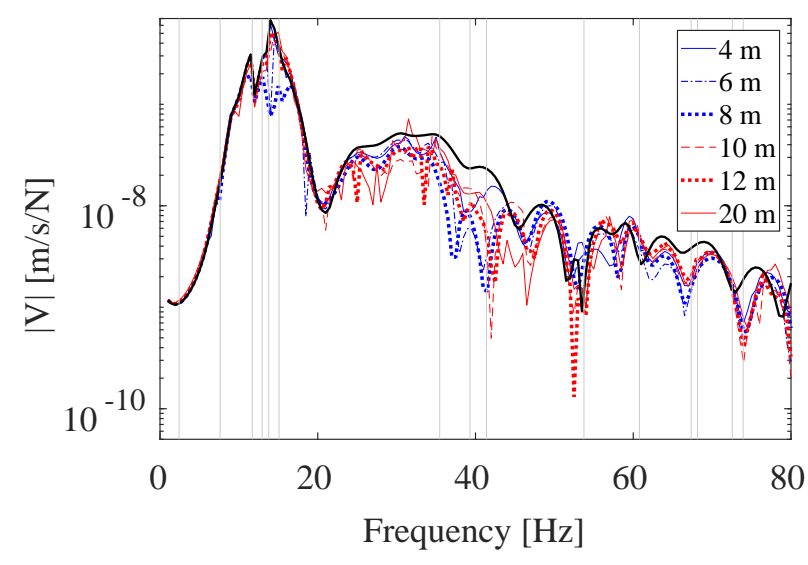

(a) Frequency spectrum for the tested values of $w$.

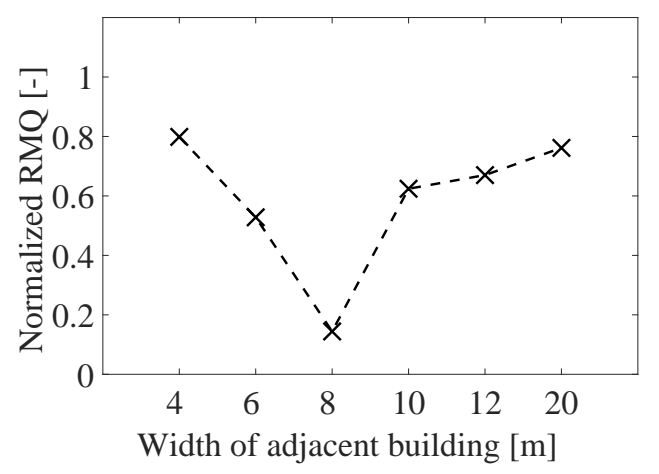

(b) RMQ value normalized to the reference case. Note the inconsistent scale on the horizontal axis.

Figure 9: Vibration levels computed with the adjacent building in front of the receiving building. The width of the adjacent building $w$ being varied. A setup with no adjacent building represents the reference case. In the left subfigure, the solid black line represents the reference case and the gray vertical lines show the receiving building's eigenfrequencies.

\subsection{Size of building footprint}

In this subsection we investigate the effects of the footprint size of the adjacent building, when located in front of the receiving building, on the receiving building's response. The model can be seen in Fig. 5, where the footprint size is varied by changing of the width $w$ of the adjacent building. As seen in Fig. 9, the footprint size can have a marked influence on the response. The most significant effect is seen when the adjacent building has the same footprint as the receiving building $(w=8 \mathrm{~m})$, where a large reduction in the vibration response is evident. In this setup, the adjacent and receiving buildings have the same modal properties.

\subsection{Cellar depth}

The influence of the cellar depth for two different locations of the adjacent building is investigated in this subsection. In the first case, the adjacent building is located in front of the receiving building as seen in Fig. 5. The distance $d$ is fixed at $15 \mathrm{~m}$, and the width of the adjacent building is $8 \mathrm{~m}$; we vary the cellar depth $h$. As seen in Fig. 10, there is a large effect of the cellar depth on the vibration response. The largest influence occurs when the cellar goes all the way down to the bedrock, i.e. $h=10 \mathrm{~m}$. The reflection of waves from the concrete cellar walls is then significant.

As was noted in Section 4.1, having the adjacent building without cellar located behind the receiving building did not, to any appreciable extent, affect the vibrational response for any of the tested distances between the buildings. However, the distance has an influence when the adjacent building has a cellar. Now we vary the cellar depth $y$ of the adjacent building located behind the receiving building. The distance $x$ is fixed at $15 \mathrm{~m}$ and the width of the adjacent building is $8 \mathrm{~m}$; see Fig. 6 for the model. As seen in Fig. 11, there is only a small effect of varying the cellar depth, but a clear trend is shown; with deeper cellar the reflected waves amplify (normalized RMQ value greater than 1) the response in the receiving building. 


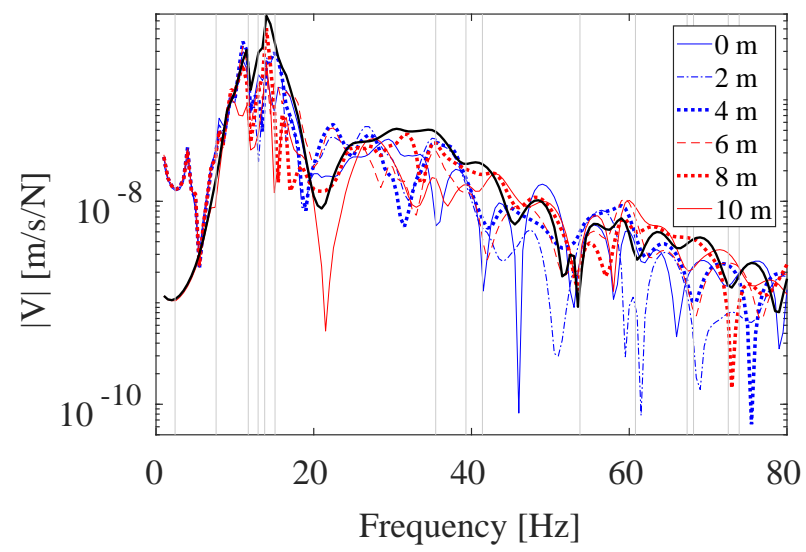

(a) Frequency spectrum for the tested values of $h$.

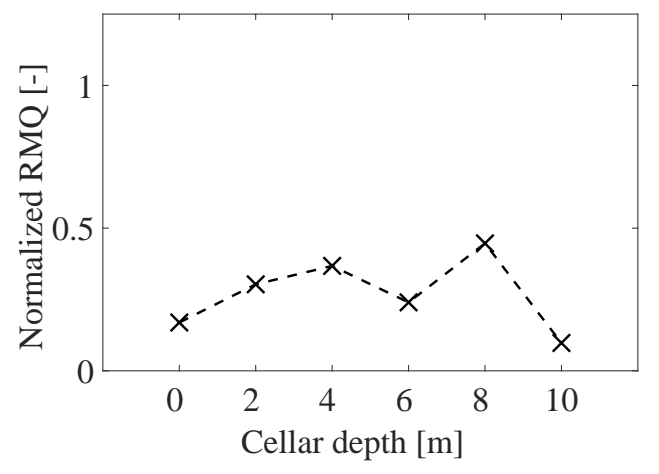

(b) RMQ value normalized to the reference case.

Figure 10: Vibration levels computed employing the adjacent building with cellar in front of the receiving building. The cellar depth $h$ being varied. A setup with no adjacent building represents the reference case. In the left subfigure, the solid black line represents the reference case and the gray vertical lines show the receiving building's eigenfrequencies.

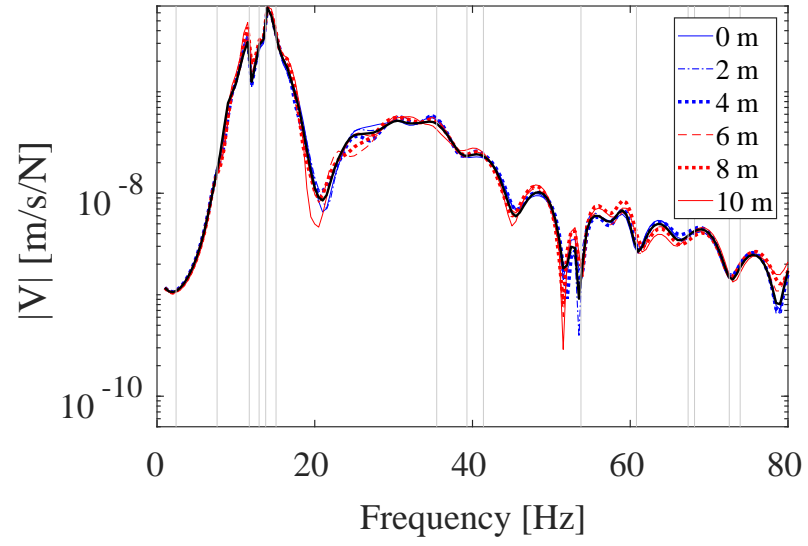

(a) Frequency spectrum for the tested cellar depths of the adja- (b) RMQ value normalized to the reference case. cent building.

Figure 11: Vibration levels computed with the adjacent building with cellar behind the receiving building. The cellar depth $y$ being varied. A setup with no adjacent building represents the reference case. In the left subfigure, the solid black line represents the reference case and the gray vertical lines show the receiving building's eigenfrequencies.

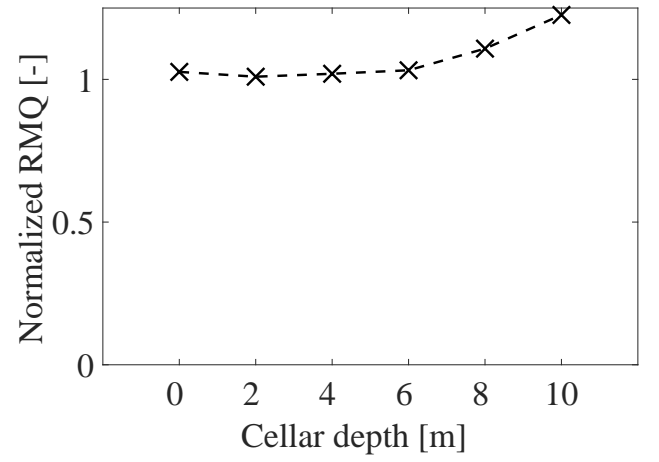




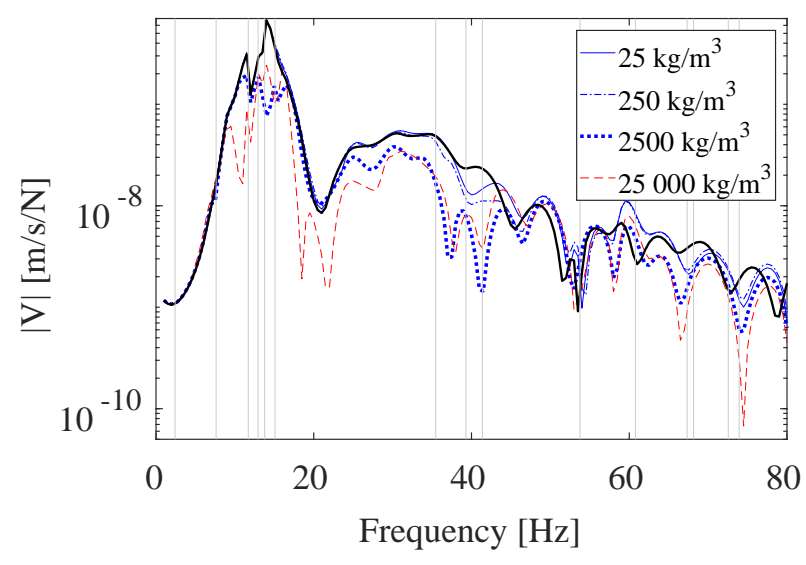

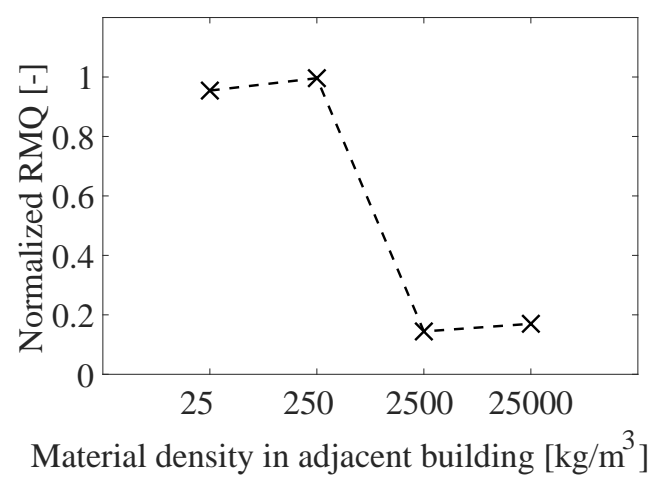

Material density in adjacent building $\left[\mathrm{kg} / \mathrm{m}^{3}\right]$

(a) Frequency spectrum for the tested density values of the ad- (b) RMQ value normalized to the reference case. jacent building.

Note the scale on the horizontal axis.

Figure 12: Vibration levels computed with the adjacent building in front of the receiving building. The mass density of the adjacent building being varied. A setup with no adjacent building represents the reference case. In the left subfigure, the solid black line represents the reference case and the gray vertical lines show the receiving building's eigenfrequencies.

\subsection{Building mass}

In this subsection we vary the mass of the adjacent building by changing the material density of the concrete. The adjacent building is located in front of the receiving building; see Fig. 5 for the model. The distance $d$ is fixed at $15 \mathrm{~m}$, the width of the adjacent building is $8 \mathrm{~m}$, and the building does not have a cellar, i.e. cellar depth $h=0$.

As seen in Fig. 12 the mass of the adjacent building has a significant effect on the response. A heavier adjacent building in front of the receiving building mitigates the vibrations to a larger extent than a lighter building. Note that the lowest as well as the highest tested mass densities are not realistic-however, they indicate that the largest influence occurs for mass densities between 250 and $2500 \mathrm{~kg} / \mathrm{m}^{3}$.

\subsection{Building damping}

The amount of damping in the adjacent building, which is located in front of the receiving building according to Fig. 5, is varied. The distance $d$ is fixed at $15 \mathrm{~m}$, and the width of the adjacent building is $8 \mathrm{~m}$ and it does not have a cellar, i.e. cellar depth $h=0$. The loss factor was varied between 0.02 and 0.2 . It was seen that varying the damping in the adjacent building had a minor effect on the evaluated vibration levels in the receiving building; see Fig. 13 for the response.

\section{CONCLUSIONS}

The paper presents a conceptual study of the effects of structural parameters of an adjacent building on the vibration response in a receiving building. The FE method was used to create a coupled soil and structure model that was excited by a ground surface load. The analysis was conducted in the frequency domain with plane strain conditions, and the velocity levels were computed in the frequency range of $1-80 \mathrm{~Hz}$ and evaluated at the midpoint of the first floor. 


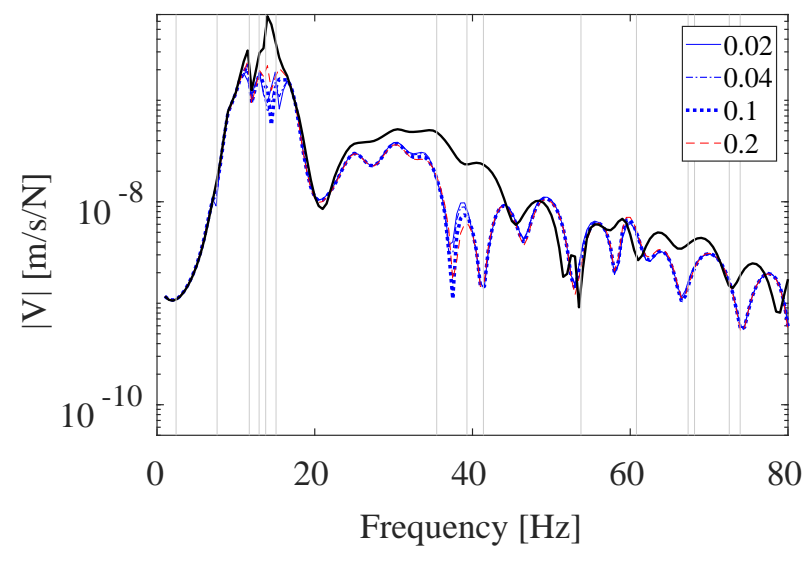

(a) Frequency spectrum for the tested damping values of the adjacent building.

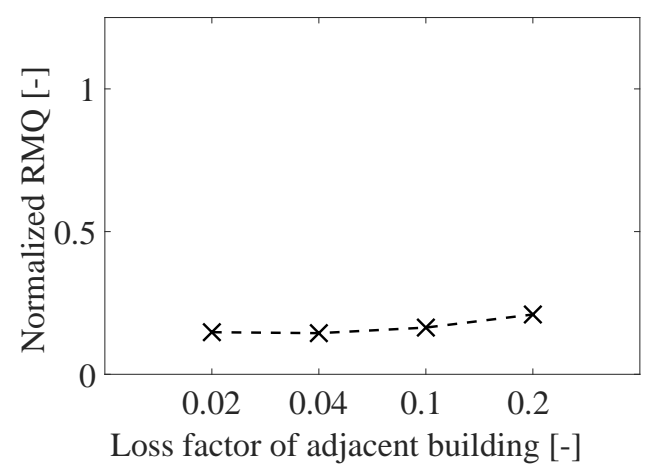

(b) RMQ value normalized to the reference case. Note the scale on the horizontal axis.

Figure 13: Vibration levels computed with the adjacent building in front of the receiving building. The amount of damping in the adjacent building being varied by use of the loss factor $\eta$. A setup with no adjacent building represents the reference case. In the left subfigure, the solid black line represents the reference case and the gray vertical lines show the receiving building's eigenfrequencies.

Two main general tendencies were observed: (i) to place a building between the vibration source and the receiving building may work as a wave obstacle, thus mitigating the incoming ground vibrations - the benefit is greater when the intervening building is heavier; (ii) having building(s) with a cellar behind the receiving building may amplify the response and should therefore be considered in a vibration analysis.

This conceptual study clearly points that there are so-called "site-city effects" in the problem of ground-surface induced building vibrations, and that adjacent buildings must be considered in an analysis aiming at determining vibrations levels in a building.

\section{ACKNOWLEDGMENTS}

The research presented in this paper was partly funded by several agencies. They being: Swedish Governmental Agency for Innovation Systems, grant ref.no. 2018-04159; The city of Helsingborg through Plattformen; and The Swedish Foundation for International Cooperation in Research and Higher Education (STINT). The financial supports are gratefully acknowledged. The former Master's student Johansson [13] is gratefully acknowledged for his contribution to the research presented in the paper.

\section{REFERENCES}

[1] A. Kham, J.F. Semblat, P.Y. Bard, P. Dangla, Seismic site-city interaction: Main governing phenomena through simplified numerical models. Bulletin of the Seismological Society of America, 96(5), 1934-1951, 2006.

[2] J. Liang, B. Han, M.I. Todovorska, D.M. Trifunac, 2D dynamic structure-soil-structure interaction for twin buildings in layered half-space I: Incident SH-waves. Soil Dynamics and Earthquake Engineering, 102, 172-194, 2017. 
[3] M. Villot, P. Ropars, P. Jean, E. Bongini, F. Poisson, Modeling the influence of structural modifications on the response of a building to railway vibration. Noise Control Engineering Journal, 59(6): 641651, 2011.

[4] L.V. Andersen, Influence of dynamic soil-structure interaction on building response to ground vibration. M.A. Hicks, R.B.J. Brinkgreve, A. Rohe eds. Numerical Methods in Geotechnical Engineering VIII, 2, pp. 1087-1092, 2014.

[5] P. Persson, K. Persson, G. Sandberg, Numerical study on reducing building vibrations by foundation improvement. Engineering Structures, 124, 361-375, 2014.

[6] P. Persson, L.V. Andersen, K. Persson, P. Bucinskas, Effect of structural design on trafficinduced building vibrations. Procedia Engineering, 199, 2711-2716, 2017.

[7] A. Clot, R. Arcos, J. Romeu, Efficient three-dimensional building-soil model for the prediction of ground-borne vibrations in buildings. Journal of Structural Engineering, 143(9), ASCE, 2017.

[8] P. Persson, L.V. Andersen, Efficient finite-element analysis of the influence of structural modifications on traffic-induced building vibrations. A.S. Cardoso, J.L. Borges, P.A. Costa, A.T. Gomes, J.C. Marques, C.S. Vieira eds. Numerical methods in geotechnical engineering IX, 2, pp. 1557-1564, 2018.

[9] R.R. Craig Jr, A.J. Kurdila, Fundamentals of structural dynamics. John Wiley \& Sons, New Jersey, 2006.

[10] J. Lysmer, R.L. Kuhlemeyer, Finite dynamic model for infinite media. Journal of Engineering Mechanics Division, 95(4), 859-877, ASCE, 1969.

[11] Dassault Systémes SIMULIA. Abaqus 6.13.

[12] International Organization for Standardization, Mechanical vibration and shock - Evaluation of human exposure to whole-body vibration - Part 1: General requirements. ISO 2631-1:1997.

[13] A. Johansson, Structural effects on externally induced building vibrations. Report TVSM5230 (Master's dissertation), Department of Construction Sciences, Lund University, 2018. 